\title{
Soon After the Birth of Radio Sources
}

\section{Owsianik}

MPIfR, Auf dem Hügel 69, 53121 Bonn, Germany

J. E. Conway, A.G. Polatidis

OSO, S-43992 Onsala, Sweden

\begin{abstract}
.
Compact Symmetric Objects (CSOs) represent an early stage in the life of radio galaxies. Our kinematic studies over a 20 year timebase have unambiguously demonstrated that they expand fast and that the radio activity is recently born. The CSO evolution with time is however still a matter of investigation. If they are progenitors of the "classical double" radio sources, then in order to fit the the observed statistics of radio sources, CSOs must have decrease their luminosity and expansion speed with time. It is also possible that CSOs represent a phase of periodic activity of the central engine; in this case the presence of the old activity in the form of extended weak emission, should be observed.
\end{abstract}

\section{Introduction}

CSOs are a class of young radio source that have symmetric parsec-scale radio jets terminating in steeper-spectrum hotspots that are embedded in radio lobes with overall size smaller than $1 \mathrm{kpc}$, and hence located in the host galaxy inside the Narrow Line Region (Wilkinson et al. 1994).

We have studied the kinematics of three such objects: $0108+388,0710+439$ and $2352+495$, using multi-epoch VLBI observations over a period of almost 20 years (from the Pearson \& Readhead (1998) VLBI survey and subsequent observations). The first results from this analysis of the $5 \mathrm{GHz}$ VLBI data were published in Owsianik \& Conway (1998), Owsianik et al. (1998) and Owsianik et al. (1999). In June 2000 we have performed new VLBA+Effelsberg $5 \mathrm{GHz}$ observations of these sources, thereby extending the time baseline to more than twenty years. To Owsianik et al.'s (1998) analysis of three epochs of $0108+388$ we have also added the VSOP data from 1999 (courtesy of W. Tschager).

Numerical parameters were estimated from the images by fitting gaussian components to the visibility data using MODELFIT in the DIFMAP package. Due to the variable data quality and image sensitivity, the core, with its highly inverted spectrum, was not unambiguously identified in all $5 \mathrm{GHz}$ images and motions relative to the core were therefore not studied. However we were able to improve our study of the separation between components as a function of time and, by fitting linear regression lines to this data, to estimate the relative 
velocities. The outermost compact components in the sources' radio structure were identified with the hotspots, based on their location and spectral indices. We have found that in all three objects the separations between hotspots increase in time with an apparent velocities of $\sim 20 \%$ of the speed of light $\left(0.18 \pm 0.01 h^{-1} c\right.$ for $0108+388,0.31 \pm 0.02 h^{-1} c$ for $0710+439$ and $0.14 \pm 0.03 h^{-1} c$ for $\left.2352+495\right)$.

Since in all CSOs, the hotspots are nearly always observed to lie close to the end of the mini-lobes in which they are embedded in, the mean expansion speed of the whole source must equal the mean expansion rate of the hotspots. Such a conclusion is consistent with CSOs being orientated close to the plane of the sky and with their hotspot-core arm-length ratio being close to one (Readhead et al. 1996). However the total source expansion could be also affected by interaction with the ISM. The narrow line region of the AGN is, very likely, highly filamentary and clumpy. The density differences can result in significantly different expansion speeds on either side of the central engine. Indeed, in the case of $0710+439$ there is indication for such an asymmetric hotspot expansion rate (Owsianik \& Conway 1998). Furthermore, in 0108+388, changes of the hotspot separation vector are seen not only in the direction parallel to the vector but also perpendicular to it (Owsianik et al. 2000, in prep).

The sub-galactic sizes of CSOs allowed us to probe the ambient medium, and invoke a density of $<5 \mathrm{~cm}^{-3}$ assuming equipartition conditions. Furthermore, we find that CSO hotspots radiate away more of the jet energy $(\sim 50 \%)$ than in FRII sources $(\sim 9 \%)$ (Owsianik 1999), which is consistent with CSOs evolving into FRIIs. However, the presence of weak extended emission in $0108+388$ (Baum et al. 1990), suggests another possibility for at least some CSOs, that the radio activity is quasi-periodic. We calculated the hotspot radiation lifetimes for the CSOs studied and found them to be only a few hundred years. Therefore the hotspots would fade away very quickly if the central engine would switch off, and previous activity periods would manifest themselves as extended steep spectrum emission, consistent with what is observed in $0108+388$.

The CSOs provide a powerful laboratory to study the physics of active galaxies and their environments in a very early stage after the activity has begun. Our studies have established that the radio activity has recently started, have determined their growth rate and have estimated parameters of their ISM environment. However, further study is required to shed light in the possible evolution paths of CSOs and to further investigate the physical conditions prevailing in them.

\section{References}

Baum, S. A., O'Dea, C. P., Murphy, D.W., de Bruyn, A.G. 1990, A\&A, 232, 19 Owsianik, I. \& Conway, J.E. 1998, A\&A 337, 69

Owsianik, I., Conway, J.E., Polatidis, A.G. 1998, A\&A, 336, L37

Owsianik, I., Conway, J.E., Polatidis, A.G. 1999, New Astr. Rev., 47, 669

Owsianik, I., PhD. Thesis, Torun Nicolaus Copernicus University, 1999

Pearson, T.J. \& Readhead, A.C.S. 1988, ApJ, 328, 14

Readhead, A.C.S., Taylor, G.B., Pearson, T.J. et al 1996, ApJ, 460, 634

Wilkinson, P.N., Polatidis, A.G., Readhead, A.C.S. et al. 1994, A\&A, 432, L87 